\title{
Clostridium Botulinum Toxins: Nature and Preparation for Clinical Use
}

\author{
J. MELLING, P. HAMBLETON and C. C. SHONE \\ Salisbury
}

\begin{abstract}
Summary
C. botulinum neurotoxins are acutely toxic materials and act by inhibiting release of the neurotransmitter acetylcholine. The specific nature of this inhibition is discussed and the preparation and purification of Type A toxin specifically for clinical use is described.
\end{abstract}

There have been few drugs whose mode and site of action and detailed pharmacology have been so well understood prior to their clinical application as Type $\mathrm{A}$ botulinum toxin. Indeed, the nature of this family of neuroparalytic agents as the most acutely potent microbial toxins and the cause of the foodborne illness botulism has made regulatory authorities particularly cautious in approving their clinical application. Nevertheless the use of the toxin particularly to treat a variety of dystonic conditions represents a most elegant, precise and safe treatment which reflects considerable credit on the pioneering work of Scott ${ }^{1}$ who first instituted the use of the toxin for human therapy.

The therapeutic, as well as the prophylactic (vaccine) use of microbial products is, of course, well established and as well as native products, especially antibiotics, also includes bacterial asparaginases used to treat acute lymphoid and lymphoblastic leukaemia in children. ${ }^{2}$ Increasingly we are also seeing human gene products such as insulin, growth hormone and interferons produced in microorganisms and used for human therapy. It is virtually certain that many other potent human regulatory proteins will also become available by this route.
Thus when it comes to pharmaceutical production $C$. botulinum toxin can be viewed as one of the first of a group of high activity protein products prepared from cultures of micro-organisms and the problems and solutions in handling such materials have general applications.

The purpose of this article is to review the nature of $C$. botulinum toxins and to describe the procedures involved in the production, purification and presentation of the type $\mathrm{A}$ toxin for clinical use.

\section{The Nature of the Toxins \\ Structure}

Complex toxins. There are eight serologically distinct toxins (A, B, C $1, C_{2}, D, E, F$ and G) produced by $C$. botulinum that are among the most powerful neuroparalytic agents known. The toxins so far studied all appear to occur both naturally and in in vitro culture complexed with non-toxic proteins which may co-purify with the toxin moiety (Fig. 1). Of these complexes the smallest, the $\mathbf{M}$ or medium complexes, comprise the neurotoxin moiety (ca. 150,000 daltons) associated with a similar sized non-toxic protein of undetermined biological activity and are the only forms found for the types $\mathrm{C}^{3}, \mathrm{E}^{4,5}$ and $\mathrm{F}^{6}$ and

From Vaccine Research and Production Laboratory, PHLS, Centre for Applied Microbiology and Research, Porton Down, Salisbury, Wiltshire.

Correspondence to: J. Melling, CAMR, Porton Down, Salisbury, Wilts SP4 0JG, UK. 
Sedimentation coefficent

Molecular weight (kdaltons)
$19 S$

900

500

300

150

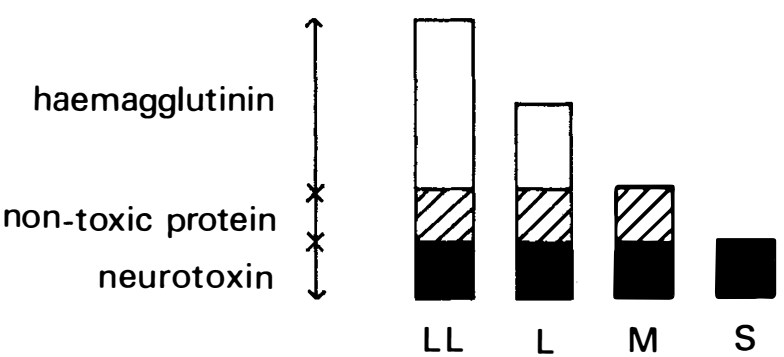

Toxin type

A

B

$\mathrm{C}_{1}$

D

E $F$

G

Complexes

LL, L,M S. L,M, S.

$M, S$.

L,M,S.

$M, S . \quad M, S$.

?

Fig. 1.

haemagglutinin-negative strains of type $\mathrm{D}^{7}$. Types $\mathrm{A}^{8}, \mathrm{~B}^{9}$ and haemagglutinin-positive $\mathrm{D}^{7}$ also occur as trimolecular or $\mathrm{L}$ (large) complexes comprising the $\mathbf{M}$ complex in association with a non-toxic protein having haemagglutinin activity. The $\mathrm{L}$ complexes (Ca, 500,000 daltons) are the largest complex states of these toxins with the exception of type A toxin which also occurs as a complex of about 900,000 daltons. This latter complex, which is thought to consist of dimers of the $\mathrm{L}$ complex, is the molecular size of the type A toxin in the crystalline state.

Neurotoxins. The various $C$. botulinum neurotoxins, although serologically distinct, appear to be structurally very similar to each other; the purified neurotoxins having similar molecular sizes and sharing a common subunit structure (Table II). These single chain progenitor toxins may be cleaved by proteolytic enzymes to give biologically active dichain toxins (Fig. 2).

The dichain toxins consist of one large (heavy, H) and one small (light, L) subunit linked by at least one disulphide bridge; the $\mathrm{H}$ subunits are about double the molecular size of the L subunits ${ }^{4.6 .10-19}$ (Table I).

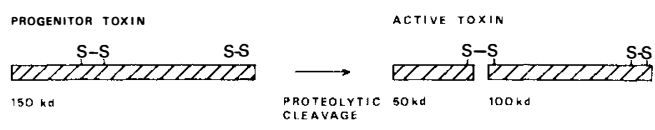

Fig. 2.
The amino acid composition of several toxin types (A, B, C, D, E and F) have been evaluated ${ }^{16.18 .20-22}$ and similarities are apparent. In particular all have high contents of hydrophobic amino acids residues which may be relevant to the membrane binding/internalisation of the toxin as discussed later. The similarities in amino acid composition together with their shared pharmacological action imply that, despite their lack of antigenic cross-reactivity, there may well be regions of structural homology within the neurotoxins.

Neurotoxin subunits. Reduction of the disulphide link(s) between $\mathrm{H}$ and $\mathrm{L}$ subunits generally leads to a loss of toxicity and a reduction in the solubility of the toxin. The subunits of reduced neurotoxin remain associated by covalent forces but these can be dissociated and solubilised by urea and subsequently separated chromatographically..$^{16-18,23-25}$

Purified $\mathrm{H}$ and $\mathrm{L}$ subunits of the neurotoxins are of greatly reduced specific toxicity $(<0.5$ per cent $)$ compared to the intact toxin; the residual toxicity probably being due to trace contamination with intact toxin.$^{16}$ Separated neurotoxin subunits appear to maintain their structural integrity and can be recombined into intact neurotoxin by mixing equimolar portions of each subunit, in reducing conditions, and slowly removing the 
Table I Molecular size and subunit structure of C. botulinum neurotoxins

\begin{tabular}{ccccc}
\hline & \multicolumn{3}{c}{ Molecular size ( $k$ daltons) } \\
\cline { 2 - 4 } Toxin type & Holotoxin & H subunit & L subunit & Reference \\
\hline A & 145 & $95-97$ & 55 & 10,11 \\
B & $155-170$ & $101-104$ & $51-59$ & $12-14$ \\
C & $141-144$ & $92-98$ & $52-53$ & $15-17$ \\
D & 140 & 85 & 55 & 18 \\
E & $147-150$ & 102 & 50 & 4 \\
F & 155 & 105 & 56 & 6,19 \\
G & Ca.150? & Not yet chracterised & \\
\hline
\end{tabular}

reducing agent. Some 30 per cent of the potential toxicities of reconstituted types $A^{23}$ and $\mathrm{B}^{24}$ toxins were regained and up to 70 per cent for type $\mathrm{C}$, toxin. ${ }^{16}$

Toxicity. When administered by the parenteral route the non-toxic proteins of the complexes appear not to play a role in toxicity, indeed, the highest specific toxicities are observed with the purified neurotoxin component (Table II). For example, the purified 145,000 dalton type A neurotoxin (S-form) has a specific toxicity about 3 fold higher than that of its L complex. In contrast, the complex toxins are more toxic than purified neurotoxins when administered orally (Table II). This difference is even more pronounced with the type B toxin where oral toxicities of the $\mathrm{S}, \mathrm{M}$ and $\mathrm{L}$ forms are in the ratio of $1 / 2 / 1,600$ respectively. ${ }^{26}$ Differences in the oral toxicities of toxins from two different type B strains of $C$. botulinum were attributed entirely to differences in the properties of the non-toxic proteins of the respective complex toxins. The enhanced oral toxicity of the complex toxins probably reflects the ability of the non-toxic proteins to protect the neurotoxin moiety from the hostile environment of the

Table II Enteral and parenteral toxicities of different molecular forms of $\mathrm{C}$. botulinum Type A toxin

\begin{tabular}{ccc}
\hline & $\begin{array}{c}\text { Intraperitoneal } \\
\text { route mouse } \\
\text { Molecular form }\end{array}$ & $\begin{array}{c}\text { Oral Route } \\
\text { mouse } L D_{50} / m g \text { protein } \\
\text { protein }\end{array}$ \\
\hline LL & $3.8 \times 10^{7}$ & $3.2 \times 10^{5}$ \\
L & $4.8 \times 10^{7}$ & $2.2 \times 10^{4}$ \\
M & $8.0 \times 10^{7}$ & $2.2 \times 10^{4}$ \\
S & $1.6 \times 10^{8}$ & $3.7 \times 10^{3}$ \\
\hline
\end{tabular}

Data calculated from Sakaguchi et al..$^{29}$ digestive tract prior to its uptake into the blood and lymphatic systems. ${ }^{27.28}$

\section{Mode of Action}

The botulinum neurotoxins act primarily on the peripheral cholinergic synapses where, by inhibiting the release of the neurotransmitter acetylcholine, they cause the widespread muscular paralysis characteristic of the fatal syndrome botulism. Studies with isolated neuromuscular junction preparations show that the action of the toxin is not mediated merely by its binding to the nerve membrane; since binding is rapid, whereas paralysis has a slower onset. ${ }^{30}$ Furthermore, in contrast to the rapid binding, stimulation of the nerve is required to induce paralysis which is temperature sensitive and requires the presence of calcium ions in the fluid bathing the neuromuscular junction..$^{31-33}$

It is now believed that at least three steps are involved in the inhibitory action of botulinum toxins. Firstly, there is a binding step, whereby the toxin attaches rapidly and avidly to the presynaptic nerve membrane. Secondly, an internalisation step in which toxin crosses the presynaptic membrane and a final step, or steps, whereby toxin inhibits the release of the neurotransmitter substance, acetylcholine.

Toxin binding. Selective, saturable binding of botulinum neurotoxins to presynaptic nerve membranes of both peripheral and central nerves has been shown using labelled toxin molecules. ${ }^{34-36}$ Neurotoxin visualised with ferritin-labelled antibody was observed to bind in discrete patches of varying size rather than uniformly over the nerve surface. ${ }^{36}$

Studies using synaptosomes from rat or mouse brain have shown that the binding of 
radio-labelled toxin is inhibited by an excess of unlabelled toxin, indicating that a limited number of binding sites are available. ${ }^{37}$ Two types of acceptor component appear to be involved; a small proportion of sites bind neurotoxin with high affinity $(\mathrm{Kd}<1 \mathrm{nM})$ and a larger pool of acceptor sites bind toxin with lower affinity (Kd>20 nM) (Table III). If one assumes that each neurotoxin contains but one binding (active) site then it seems most likely that the toxin forms a 'good fit' with the high affinity sites but a 'poor fit' with the low affinity ones.

Table III Binding constants for high and low affinity synaptosomal acceptor sites for botulinum toxins

\begin{tabular}{cllc} 
& \multicolumn{2}{c}{$K d(n M)$} & \\
\cline { 2 - 3 } Toxin type & High & Low & Reference \\
\hline A & 0.6 & 25 & 37 \\
B & $0.3-0.5$ & 20 & 14 \\
C & 0.08 & 35 & 38,39 \\
D & 0.023 & 35 & 18 \\
\hline
\end{tabular}

Although the similar structural and pharmacological properties of the various botulinum neurotoxin types suggest they may have similar modes of action, it would appear that the synaptosomal acceptor sites may differ from some toxin types. There may, indeed, be at least two groups of synaptosomal acceptors, one recognised by the $\mathrm{A}$ and $\mathrm{E}$ toxins and one by the Type B toxin, since there is little or no competition between Types $\mathrm{A}$ and $\mathrm{B}$ or $\mathrm{B}$ and $\mathrm{E}$ for binding to synaptosomes whereas Types $\mathrm{A}$ and $\mathrm{E}$ appear to compete for the same acceptor sites. ${ }^{14,37,40}$ Similarly, binding of Type D toxin is completely inhibited by $\mathrm{C}_{1}$ toxin, suggesting common acceptor components. ${ }^{18}$ Whether these are similar to the acceptors for either $\mathrm{A}$ and $\mathrm{E}$ or B toxins is not yet known.

The role of subunits in binding to acceptors. In those botulinum neurotoxins so far studied the $\mathrm{H}$ subunit appears to contain the active site which binds to synaptosomal acceptor. For both $\mathrm{A}$ and $\mathrm{B}$ toxins homologous unlabelled $\mathrm{H}$-subunit reduces binding of radio-labelled toxin, whereas the $\mathrm{L}$ chain is without effect. Similarly Type D toxin binding is inhibited by Types $\mathrm{C}$, and $\mathrm{D} \mathrm{H}$ subunits.
The single chain forms of B and E toxins bind to synaptosomes with similar affinities to nicked, active toxin ${ }^{14,42}$ implying that the relative non-toxicity of the single chain molecule does not reflect an inability to bind to the nerve terminal but rather to some subsequent step in the toxins' action.

Direct evidence of toxin internalisation was provided by experiments using hemidiaphragms incubated with radio-labelled Type A neurotoxin. ${ }^{34}$ Electron micro-autoradiographs showed that following binding to the nerve terminal membrane transfer of labelled toxin across the plasma membrane was evident after about 20 minutes and was maximal after 90 minutes. The translocation process which did not require the presence of $\mathrm{Ca}^{2+}$ ions nor indirect stimulation of the nerve was abolished at low temperatures or by inhibitors of energy production. The latter did not, however, prevent binding to the membrane surface indicating that energy is required only for internalisation. From electron micrographs of nerve preparations in which toxin internalisation was prevented it was estimated that there were between 150500 acceptor sites per $\mu \mathrm{m}^{2}$ on the nerve surface. ${ }^{34}$ Detailed quantitation of EM autoradiographs revealed that not all bound material was internalised and that some 60 per cent remained associated with the membrane. Neurotransmitter release. Since botulinum toxins appear to inhibit neither neurotransmitter synthesis nor storage disruption of the $\mathrm{Ca}^{2+}$-mediated acetylcholine release mechanism seems probable. Three possibilities exist; toxin could inhibit $\mathrm{Ca}^{2+}$ influx during nerve stimulation or stimulate $\mathrm{Ca}^{2+}$ efflux, or it could act directly on some stage in the release mechanism itself. No hypothesis adequately accounts for all the available data although most evidence argues against botulinum toxin acting by inhibiting $\mathrm{Ca}^{2+}$ influx.

In the early stages of botulinum toxin poisoning of the neuromuscular junction the effects can be reversed by increasing the flow of $\mathrm{Ca}^{2+}$ into the nerve terminal. ${ }^{43}$ However, as the poisoning progresses so the ability of $\mathrm{Ca}^{2+}$ to reverse the toxic effects is diminished. It is conceivable then, that the botulinum toxin may act by reducing the sensitivity of the neurotransmitter release mechanism to $\mathrm{Ca}^{2+}$ such 
that the normal intracellular $\mathrm{Ca}^{2+}$ levels are insufficient to promote release.

Similar conclusions are indicated by studies with substances such as tetraethylammonium $^{43}$ and 4-aminopyridine ${ }^{44-46}$ which, in prolonging the depolarisation/repolarisation cycle of the nerve, allow higher concentrations of $\mathrm{Ca}^{2+}$ to build up in the nerve terminal. In these conditions the end plate potentials of mildly botulinum-poisoned neuromuscular junction preparations can be restored to normal.

Many key aspects of the action of the botulinum neurotoxins still await clarification, including the appearance of minature end plate potentials shortly after the onset of poisoning and particularly the long duration of the toxic effects. One possibility is that the toxin evokes a permanent change in an enzyme, or other vital system, having a long half life. ${ }^{47.79}$ Indeed it has been proposed that, in common with some other microbial toxins, the toxin itself may be an enzyme; the protracted action and high specificity of the toxin appears to support this view.

\section{Preparation of Type A Toxin for Clinical Use} For a product to be acceptable and licensable for clinical use appropriate standards of quality, safety and efficacy must be achieved. Thus material must be reproducible in its composition and potency from batch to batch and stable during a particular period under defined and achievable storage conditions. Safety and efficacy need to be assessed both by animal experimentation and in human trials and to be verifiable on subsequent batches by the application of relevant quality control tests. All of the above requires the application of Good Manufacturing Practice (GMP) techniques. GMP builds on laboratory biochemistry and microbiology and translates a Research and Development process into an effective pharmaceutical operation. The importance of standardising conditions for production and purification is clearly apparent given the effect of proteolytic 'nicking' on toxic activity and the further susceptibility of the protein toxin to inactivation by proteolytic enzymes.

Culture. Cultures of $C$. botulinum from a verified seed stock are grown up and inoculated into a 301 fermenter operated under anaerobic conditions and toxin production and other culture parameters are monitored (Fig. 3). When the maximum yield of toxin has been attained $\left(2 \times 10^{6}\right.$ mouse $\left.\mathrm{LD}_{50} / \mathrm{ml}\right)$ usually after 72 hours the toxin is harvested by centrifugation after acidifying the culture. In this form the toxin can be stored prior to purification. Cultures are extensively checked for toxin activitiy, identity and absence of contamination.

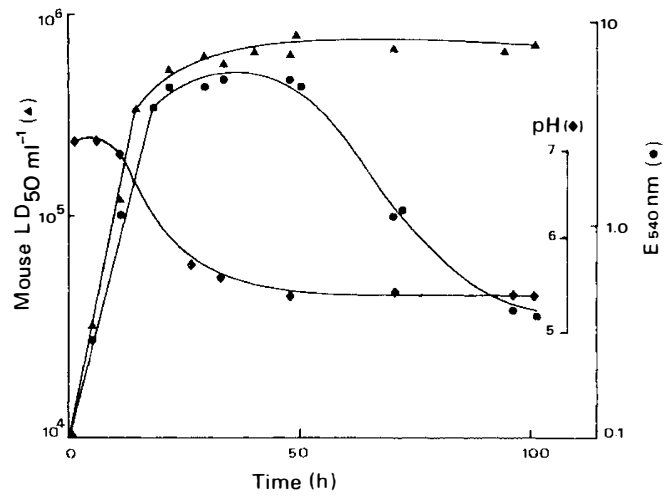

Fig. 3 .

Purification. The precipitated crude toxin is re-dissolved and purified by a series of procedures involving ammonium sulphate precipitation and ion-exchange chromatography (Table IV). During these procedures the process is monitored for the absence of contamination with extraneous micro-organisms as well as the toxicity and protein content of the extracts.

Formulation and Freeze Drying. The potency of the purified toxin is assessed and an appropriate quantity of the purified toxin solution is added to a diluent containing lactose and human serum albumin. The diluent is designed to provide protection for the toxin during freeze-drying and to act as a bulking agent for the freeze dried product. Prior to freeze drying, the diluted toxin is dispensed into vials and at the completion of the drying process vials are checked for integrity, sterility, moisture content and potency.

\section{Clinical Use and Dosage}

C. botulinum toxin has now been used to treat both experimentally and routinely a range of 
Table IV Purification of C. botulinum Type A Hemagglutinin-neurotoxin Complex ${ }^{48}$

\begin{tabular}{|c|c|c|c|c|c|}
\hline \multirow[b]{3}{*}{ Stage procedure } & \multirow{3}{*}{$\begin{array}{l}\text { Protein } \\
(m g)\end{array}$} & \multicolumn{4}{|c|}{ Toxicity } \\
\hline & & \multirow{2}{*}{$\begin{array}{c}\text { Total } \\
\text { mouse } \\
L D_{s 0}\end{array}$} & \multirow{2}{*}{$\begin{array}{l}M L D_{s_{0}} / m g \\
\text { protein }\end{array}$} & \multicolumn{2}{|c|}{ Recovery \% } \\
\hline & & & & Stage & Overall \\
\hline 1 Whole culture (20.1) & - & $10^{10}$ & - & 100 & 100 \\
\hline 2 Precipitation at $\mathrm{pH} 3.5$, and adjusted with $3 \mathrm{~N}$ & & & & & \\
\hline $\mathrm{H}_{2} \mathrm{SO}_{4}$ & 4,600 & $7.5 \times 10^{9}$ & $1.6 \times 10^{6}$ & 75 & 75 \\
\hline 3 Extraction with $0.2 \mathrm{M}$ phosphate buffer at & & & & & \\
\hline pH 6.0 & 1,300 & $6.8 \times 10^{9}$ & $5.2 \times 10^{6}$ & 91 & 68 \\
\hline $\begin{array}{l}4 \text { Ribonuclease treatment }\left(100 \mathrm{ug} / \mathrm{ml}, 34^{\circ}, 3 \mathrm{~h}\right) \\
5 \text { Precipitation at } 60 \text { per cent saturation }\left(2 t 25^{\circ}\right)\end{array}$ & 1,300 & $6.8 \times 10^{9}$ & $5.2 \times 10^{6}$ & 100 & 68 \\
\hline $\begin{array}{l}5 \text { Precipitation at } 60 \text { per cent saturation (at } 25^{\circ} \text { ) } \\
\text { of ammonium sulphate }\end{array}$ & - & $65 \times 10^{9}$ & - & 96 & 65 \\
\hline 6 DEAE-Sephadex A50 batch preabsorption & 860 & $5.0 \times 10^{9}$ & $5.8 \times 10^{6}$ & 77 & 50 \\
\hline $\begin{array}{l}7 \text { DEAE-Sephacel ion-exchange chromato- } \\
\text { graphy at pH } 5.5\end{array}$ & 195 & $4.8 \times 10^{9}$ & $2.5 \times 10^{7}$ & 96 & 48 \\
\hline $\begin{array}{l}8 \text { Precipitation at } 60 \text { per cent saturation (at } 25^{\circ} \text { ) } \\
\text { if ammonium sulphate }\end{array}$ & 195 & $4.8 \times 10^{9}$ & $2.5 \times 10^{7}$ & 100 & 48 \\
\hline
\end{tabular}

conditions where deliberate paralysis of a particular muscle may be of benefit. The conditions include strabismus, ${ }^{1}$ blepharospasm, ${ }^{49}$ lateral rectus paresis, ${ }^{50}$ hemifacialspasm, ${ }^{51}$ Meige syndrome,${ }^{52}$ facial synkinesis after facial nerve palsy and spasmodic torticollis..$^{53}$

Clearly the muscle sizes, and most critically the number of cholinergic synapses, vary considerably among these conditions as it is clear does the dose of toxin required for effective action. It is likely that for the effective treatment of patients and the convenience of clinicians toxin will need to be made available in a range of doses appropriate to the particular condition and even for one condition individual patient responses may dictate varying doses. Accordingly we decided to present the toxin which has been used for blepharospasm and strabismus treatment as a weight of active protein determined by mouse potency; the use of units of activity based on human physiological activity may follow as more clinical data become available.

The stability of the toxin preparation over time is clearly critical to building up an effective clinical data base and we are able to confirm that our freeze-dried toxin retains stability even when kept at $4^{\circ} \mathrm{C}$ for not less than 3 years. Thus the potency of the preparation is a constant factor even when used in different clinics, countries and over a long time period and should enable a consistent dose regime to be established for a range of dystonia conditions.
As the data presented above show, not only is the mode and site of action of the toxin highly specific but it also possesses a further important characteristic which is its avidity for the site of action. Taken together these characteristics allow local injection into a muscle with little or no general dissemination. It will eventually be interesting to investigate the clinical use of the pure $150,000 \mathrm{MW}$ neurotoxin as this molecule which does not have the haemagglutinin moiety of the complex toxin may give even better precision and reproducibility of action.

Further development may be required if there is evidence of production of antibody by patients receiving toxin. Should this become a problem it would be necessary to make available other of the serologically distinct toxin Types $B$ through $G$ and the information currently being pursued on the structure and activity of these other toxins should prove invaluable in assessing their suitability for clinical use.

\section{References}

${ }^{1}$ Scott AB, Rosenbaum AL, Collins CC: Pharmacological weakening of extra ocular muscles. Invest Ophthalmol 1973, 12: 924-7.

${ }^{2}$ Report of Working Party on Leukaemia in Childhood: Improvement in treatment for children with acute lymphoblastic leukaemia. Lancet 1986, 1: 408.

${ }^{3}$ Syuto B and Kubo S: Purification and chrystallisation of Clostridium botulinum type C toxin. Jpn J Vet Res 1972. 20: 19-30.

${ }^{4}$ Kitamura M, Sakaguchi S, Sakaguchi G: Purification 
and some properties of Clostridium botulinum type E toxin. Biochem Biophys Acta 1968, 168: 207-17.

${ }^{5}$ Kitamura M, Sakaguchi S, Sakaguchi G: Significance of $12 \mathrm{~S}$ toxin of Clostridium botulinum type E. J Bacteriol 1968, 98: 1173-8.

${ }^{6}$ Ohishi I and Sakaguchi G: Purification of Clostridium botulinum type $\mathrm{F}$ progenitor toxin., Appl Microbiol 1974, 28: 923-8.

${ }^{7}$ Miyazaki S, Iwasaki M, Sakaguchi G: Clostridium botulinum type D toxin: purification, molecular structure, and some immunological properties. Infect Immun 1977, 17: 395-401.

* Sugii S and Sakaguchi G: Molecular construction of Clostridium botulinum type A toxins. Infect Immun 1975, 12: 1262-70.

${ }^{9}$ Kazaki S, Sakaguchi S, Sakaguchi G: Purfication and some properties of progenitor toxins of Clostridium botulinum type B. Infect Immun 1974, 10: 750-6.

10 Shone CC, Hambleton P, Melling J: Inactivation of Clostridium botulinum type A neurotoxin by trypsin and purification of two tryptic fragments. Proteolytic action near the COOH-terminus of the heavy subunit destroys toxin binding activity. Eur J Biochem 1985, 151: 75-82.

$"$ Tse CK, Dolly JO, Hambleton P, Wray D, Melling J: Preparation and characterisation of homogenous neurotoxin type A Clostridium botulinum. Its inhibitory action on neural release of acetylcholine in the absence and presence of bungarotoxin. Eur J Biochem 1982, 122: 493-500.

${ }^{12}$ Beers WH and Reich E: Isolation and characterisation of Clostridium botulinum type B toxin. J Biol Chem 1969, 244: 4473-9.

${ }^{13}$ DasGupta BR and Sugiyama H: Comparative sizes of botulinum type A and B neurotoxins. Toxicon 1977, 15: 357-63.

${ }^{14}$ Evans GM, Williams RS, Shone CC, Hambleton P, Melling J, Dolly JO: Botulinum type B. Its purification, radioidination and interaction with rat-brain synaptosomal membranes. Eur J Biochem 1986, 154: 409-16.

${ }^{15}$ Syuto B and Kubo S: Isolation and molecular size of Clostridium botulinum type C toxin. Appl Environ Microbiol 1977, 33: 400-5.

${ }^{16}$ Syuto B and Kubo S: Separation and characterisation of heavy and light chains from Clostridium botulinum type $\mathrm{C}$ toxin and their reconstitution. $J$ Biol Chem 1981, 256: 3712-17.

17 Terajima J, Syuto B, Ochanda JO, Kubo S: Purification and characterisation of neurotoxin produced by Clostridium botulinum Type C 6813. Infect Immun 1985, 48: 312-17.

${ }^{18}$ Murayama S, Syoto B, Oguma K, Iida H, Kubo S: Comparison of Clostridium botulinum toxin types $\mathrm{D}$ and $\mathrm{C} 1$ in molecular property, antigenicity and binding ability to rat-brain synaptosomes. Eur J Biochem 1984, 142: 487-92.

${ }^{19}$ Yang KH and Sugiyama H: Purificaiton and properties of Clostridium botulinum type F toxin. Appl Microbiol 1975, 29: 598-603.

${ }^{20}$ DasGupta BR and Sathyamoorthy V: Purification and amino acid composition of type $\mathrm{A}$ botulinum neurotoxin. Toxicon 1984, 22: 415-24.

${ }^{21}$ DasGupta BR and Rasmussen S: Purification and amino acid composition of type $E$ botulinum neurotoxin. Toxicon 1983, 21: 535-45.

22 DasGupta BR and Rasmussen S: Amino acid composition of Clostridium botulinum type $\mathrm{F}$ neurotoxin. Toxicon 1983, 21: 566-9.

${ }^{23}$ Kozaki S, Togashi S, Sakaguchi G: Separation of Clostridium botulinum type A derivative toxin into two fragments. Jap J Med Sci Biol 1981, 34: 61-8.

${ }^{24}$ Kozaki S, Miyazaki S, Sakaguchi G: Development of antitoxin with each of two complementary fragments of Clostridium botulinum type B derivative toxin. Infect Immun 1977, 18: 761-6.

${ }_{25}$ Sathyamoorthy V and DasGupta BR: Separation, purification, partial characterisation and comparison of the heavy and light chains of botulinum neurotoxin types A, B and E. J Biol Chem 1985, 260: 10461-6.

${ }_{26}$ Ohishi I, Sugii S, Sakaguchi G: Oral toxicities of Clostridium botulinum toxins in response to molecular size. Infect Immun 1977, 16: 107-9.

${ }^{27}$ Sugii S, Ohishi I, Sakaguchi G: Correlation between oral toxicity and in vitro stability of Clostridium botulinum type A and B toxins of different molecular sizes. Infect Immun 1977, 16: 910-14.

${ }^{28}$ Sugii S, Ohishi I, Sakaguchi G: Intestinal absorption of botulinum toxins of different molecular sizes in rats. Infect Immun 1977, 17: 491-6.

${ }^{24}$ Sakaguchi G, Kozaki S, Ohishi I: Structure and function of botulinum toxins. In Bacterial Protein Toxins. Ed JE Alouf, EJ Fehrenbach, JHFreer, J Jeljiaszewicz. Academic Press, London, 1984, pp 433-43

${ }^{30}$ Burgen AS, Dickens VF, Zatman LJ: The action of botulinum toxin on the neuromuscular junction. J Physiol (London) 1949, 109: 10-24.

"Simpson LL: Ionic requirements for the neuromuscular blocking action of botulinum toxin: implications with regard to synaptic transmission. Neuropharmacol 1971, 10: 673-84.

32 Simpson LL: The interaction between divalent cations and botulinum toxin type $\mathrm{A}$ in the paralysis of the rat phrenic nerve-hemidiaphragm preparation. Neuropharmacol 1973, 12: 165-76.

33 Thesleff S: Supersensitivity of skeletal muscle produced by botulinum toxin. J Physiol (Lond) 1960 , 151: 598-607.

${ }^{34}$ Dolly JO, Black J, Williams RS, Melling J: Acceptors for botulinum neurotoxin reside on motor nerve terminals and mediate its internalisation. Nature 1984, 307: 457-60.

${ }^{35}$ Dolly JO, Williams RS, Black JD, Tse CK, Hambleton P, Melling J: Localisation of sites for 125-I labelled botulinum neurotoxin at the murine neuromuscular junction and its binding to rat brain synaptosomes. Toxicon 1982, 20: 141-8.

${ }^{36}$ Hirokawa $\mathbf{N}$ and Kitamura $\mathrm{M}$ : Binding of Clostridium botulinum neurotoxin to the presynaptic membrane in the central nervous system. J Cell Biol 1979, 81: 43-9. 
37 Williams RS, Tse CK, Dolly JO, Hambleton P, Melling $\mathrm{J}$ : Radioiodination of botulinum neurotoxin type A with retention of biological activity and its binding to rat brain synaptosomes. Eur J Biochem 1983, 131: 437-45.

${ }^{38}$ Agui T, Syuto B, Oguma K, Iida $\mathrm{H}$ and Kubo S: Binding of Clostridium Botulinum type $\mathrm{C}$ neurotoxin to rat brain synaptosomes. $J$ Biochem 1983, 94: 521-7.

${ }^{39}$ Agui T, Syuto B, Oguma K, Iida H, Kubo S: The structural relation between the antigenic determinants to monoclonal antibodies and binding sites to rat brain synaptosomes and GT1b ganglioside in Clostridium botulinum type $\mathrm{C}$ neurotoxin. J Biochem 1985, 97: 213-8.

${ }^{40}$ Kozaki S: Interaction of botulinum type A, B and E derivative toxins, with synaptosomes of rat brain. Naunyn-Schmeideberg's Arch Pharmacol 1979, 308: 67-70.

${ }^{41}$ Dreyer F, Becker C, Bigalke H, Funk J, Penner R, Rosenberg F, Ziegler M: Action of botulinum A toxin and tetanus toxin on synaptic transmission. J Physiol (Paris) 1984, 79: 252-258.

${ }^{42}$ Kozaki S and Sakaguchi G: Binding to mouse brain synaptosomes of Clostridium botulinum type E derivative toxin before and after tryptic activation. Toxicon 1982, 20: 841-6.

${ }^{43}$ Cullcandy SG, Lundh H, Thesleff S: Effects of botulinum toxin on neuromuscular transmission in the rat. J Physiol (London) 1976, 260: 177-203.

${ }^{4}$ Simpson LL: Pharmacological studies on the subcellular site of action of botulinum toxin type A. $J$
Pharmacol Exp ther 1978, 206: 661-9.

${ }^{45}$ Lundh H, Cullcandy SG, Leander S, Thesleff. S: Restoration of neurotransmitter release in the botulinum-poisoned skeletal muscle. Brain Res 1976, 110: 194-8.

${ }^{46}$ Lundh $\mathrm{H}$ and Thesleff S: The mode of action of 4-aminopyridine and guanidine on transmitter release from motor nerve terminals. Eur J Pharmacol 1977, 42: 411-12.

${ }^{47}$ Simpson LL: The origin, structure and pharmacological activity of botulinum toxin. Pharmacol Rev 1981, 33: 155-88.

${ }^{48}$ Hambleton P, Capel B, Bailey N, Heron N, Crooks A, Melling J: Production, purification and toxoiding of Clostridium botulinum type A toxin. In Biomedical Aspects of Botulism (Lewis, GE, Ed), Academic Press 1981, 247-60.

${ }^{49}$ Shorr N, Seiff SR, Kopelman J: The use of botulinum toxin in blepharospasm. $A m \quad J$ Ophthalmol 1985, 99: 542-6.

${ }^{50}$ Scott $\mathrm{AB}$ and Kraft SP: Botulinum toxin injection in the management of lateral rectus paresis. Ophthalmology 1985, 92: 676-83.

${ }^{5 l}$ Savino PJ, Sergott RC, Bosley TM, Schatz NJ: Hemifacial spasm treated with botulinum A toxin injection. Arch Ophthalmol. 1985, 103: 1305-6.

52 Mauriello JA: Blepharospasm, Meige syndrome and hemifacial spasm treated with botulinum toxin. Neurology 1985, 35: 1499-500.

${ }^{53}$ Tsui JKC, Eisen A, Stoessl AJ, Calne S, Calne DB: Double-blind study of botulinum toxin in spasmodic torticollis. Lancet 1986. ii: 245-7. 